\title{
Development of a nutritional index to evaluate the effectiveness of total parenteral nutrition during the early postoperative period after pancreaticoduodenectomy
}

\author{
Sung Whan $\mathrm{Cha}^{1}$, Sung Hyun $\mathrm{Kim}^{2} \wedge$, Song Ee Baek ${ }^{3} \wedge$, Kyung Sik Kim ${ }^{2 \wedge}$ \\ ${ }^{1}$ Department of Surgery, Yonsei University Wonju College of Medicine, Wonju, Kangwon-do, South Korea; ${ }^{2}$ Department of Surgery, Yonsei \\ University College of Medicine, Seoul, South Korea; ${ }^{3}$ Department of Radiology, Yonsei University College of Medicine, Seoul, South Korea \\ Contributions: (I) Conception and design: KS Kim; (II) Administrative support: KS Kim; (III) Provision of study materials or patients: SW Cha; (IV) \\ Collection and assembly of data: SW Cha; (V) Data analysis and interpretation: SW Cha; (VI) Manuscript writing: All authors; (VII) Final approval \\ of manuscript: All authors. \\ Correspondence to: Kyung Sik Kim, MD, PhD. Department of Surgery, Yonsei University College of Medicine, Ludlow Faculty Research Building, \\ 50-1 Yonsei-ro, Seodaemun-gu, Seoul, South Korea. Email: kskim88@yuhs.ac.
}

Background: Malnutrition leads to adverse effects on the short- and long-term prognosis in patients with periampullary diseases who underwent surgery. Nutritional risk indicators based on albumin and body weight have been developed to evaluate nutritional status and nutritional therapy efficacy, but no standard objective measurement has been devised to evaluate nutritional status during the early period after pancreaticoduodenectomy (PD). Therefore, this study aimed to assess the efficacy of total parenteral nutrition (TPN) during the early postoperative period after PD.

Methods: We analyzed 28 patients with a periampullary disease-common bile duct cancer, ampulla of Vater cancer, pancreatic head cancer, neuroendocrine tumor, chronic pancreatitis-who have undergone PD from Jan. 1, 2012 to Dec. 31, 2016. For all the patients, TPN was administered from postoperative day (POD) 1 at $25 \mathrm{Kcal} / \mathrm{kg}$ ideal body weight. Various nutritional indicators were measured such as Body mass index, nutritional risk index, protein, albumin, prealbumin, C-reactive protein. The volume of skeletal muscle area, muscle density, visceral and subcutaneous fat areas were assessed two times, preoperatively and on POD 7 by CT scan at the 3rd lumbar spine (L3) level.

Results: Average age of the 28 study subjects (18 males and 10 females) was $63.5 \pm 9.7$ years. Although there is no difference in BMI between preoperative result and POD 7, protein, albumin, and prealbumin levels were significantly lower POD 7 the preoperative $(\mathrm{P}<0.001)$, but $C R P$ was higher $(\mathrm{P}<0.001)$, and prealbumin and CRP levels were negatively correlated $(\mathrm{R}=-0.682, \mathrm{P}<0.01)$. Muscle mass increased postoperatively $(\mathrm{P}=0.02)$, but the amount of visceral fat decreased $(\mathrm{P}=0.00)$. Based on $\mathrm{CRP}$, and muscle density results, muscle, visceral and subcutaneous fat masses did not change after PD.

Conclusions: In order to evaluate the nutritional status accurately after the hepato-biliary radical surgery, we suggest the muscle and fat mass measurement that can adjust the degree of inflammation during the early postoperative period.

Keywords: Pancreaticoduodenectomy (PD); total parenteral nutrition (TPN); nutritional assessment; sarcopenia

Submitted Mar 24, 2020. Accepted for publication May 13, 2021.

doi: 10.21037 /gs-20-390

View this article at: https://dx.doi.org/10.21037/gs-20-390

^ ORCID: Sung Hyun Kim, 0000-0001-7683-9687; Song Ee Baek, 0000-0001-8146-2570; Kyung Sik Kim, 0000-0001-9498-284X. 


\section{Introduction}

Since its introduction by Whipple in 1935 (1), pancreaticoduodenectomy (PD) remains the treatment of choice for periampullary carcinomas, including such as those of the pancreas head, ampulla of Vater, duodenum, and distal common bile duct, and some benign lesions. After PD, patients are at high risk of malnutrition, because of gastrointestinal tract changes that cause poor oral intake, and loss of duodenum, which is required for nutrient breakdown and absorption $(2,3)$.

Malnutrition is an independent predictor of poor outcomes in patients who have undergone radical surgery for malignant hepatobiliary disease and increases morbidity, mortality, length of stay, and financial burden $(4,5)$. To overcome this physiologic condition, which is the hallmark of the postsurgical state, the administration of high caloric intake during the immediate postoperative period is necessary. Furthermore, the early detection of nutritional risk facilitates early intervention and prevents later complications, but no single gold standard objective measurement has been devised for the evaluation of nutritional status during the early period after radical surgery.

Nutritional support is performed routinely via the oral route, but when this is problematic, alternative sources of nutritional supplementation such as intravenous total parenteral nutrition (TPN) are essential (6). Nutritional support strategies have evolved considerably over the past decades and commonly TPN has been used postoperatively (7).

Although TPN is an effective method of nutritional support, its use is associated with risks of complications such as central line-associated bloodstream infection, hyperglycemia, liver function abnormalities, and electrolyte imbalance (8). Despite these shortcomings, TPN still plays a key role in the rescue of patients from severe postoperative complications and the risk of postoperative malnutrition. Notably, patients who have not received sufficient nutritional support for more than 14 days have remarkably elevated complications and mortality rates (9), and furthermore, malnutrition can result in sarcopenia. Thus, it is positively necessary to identify appropriate indications for early nutritional supplementation to avoid these poor outcomes.

Several types of serologic markers (e.g., albumin, transferrin, prealbumin) have been widely used to evaluate nutritional status, and shown to be associated with the prognoses of malnourished patients (10,11). Malnutrition can cause sarcopenia which is the degenerative loss of skeletal muscle mass, quality, and strength. Several techniques, such as anthropometry, bioelectrical impedance analysis (BIA), dual-energy X-ray absorptiometry (DEXA), and CT/MRI, are available to measure muscle mass. Of these tools, a CT scan provides the most valid and precise means of measuring muscle mass and fat and can measure the most exact physical properties of muscle mass (12).

However, no tool has been developed to determine the efficacy of TPN administered to patients during the early postoperative period. We aimed to assess the effectiveness of TPN for the patients who are in the early postoperative period after PD.

We present the following article in accordance with the STROBE reporting checklist (available at http://dx.doi. org/10.21037/gs-20-390).

\section{Methods}

This retrospective study was conducted at a single university hospital. We analyzed 28 patients with a periampullary disease, that underwent PD from January 1, 2012 to December 31, 2016. All patients were operated upon by a single surgeon using the same procedures, which included (I) single-loop pancreatic and biliary reconstruction; (II) pancreaticojejunostomy; (III) duct-to-mucosa anastomosis; (IV) internal pancreatic duct silicone stent placement, and (V) continuous suturing of pancreatic parenchyma to jejunal serosa with prolene.

For all the patients, TPN was administered from postoperative day (POD) 1. The total required calorie was calculated at $25 \mathrm{Kcal} / \mathrm{kg}$ ideal body weight as this calorie furnishes an approximate estimate of daily energy expenditure and requirements (13). The initial target calorie that TPN provides on POD 1 was to supply $60 \%$ of the total required calories. Then, the target calorie gradually increased daily; $80 \%$ on POD 2, and $100 \%$ on POD 3.

CT scan was taken in all the patients on POD 7 . And enteral feeding began when the CT scan showed no evidence of complications. The amount of TPN administration was gradually decreased depending on the amount of oral intake. If the patients are tolerable with $20 \%$ amount of usual oral intake, the total calorie of TPN was decreased to $80 \%$ of required calorie. This gradual increase and decrease method of TPN administration was determined by our team.

Several nutritional indicators-body mass index (BMI), nutritional risk index (NRI), protein, albumin, prealbumin, 

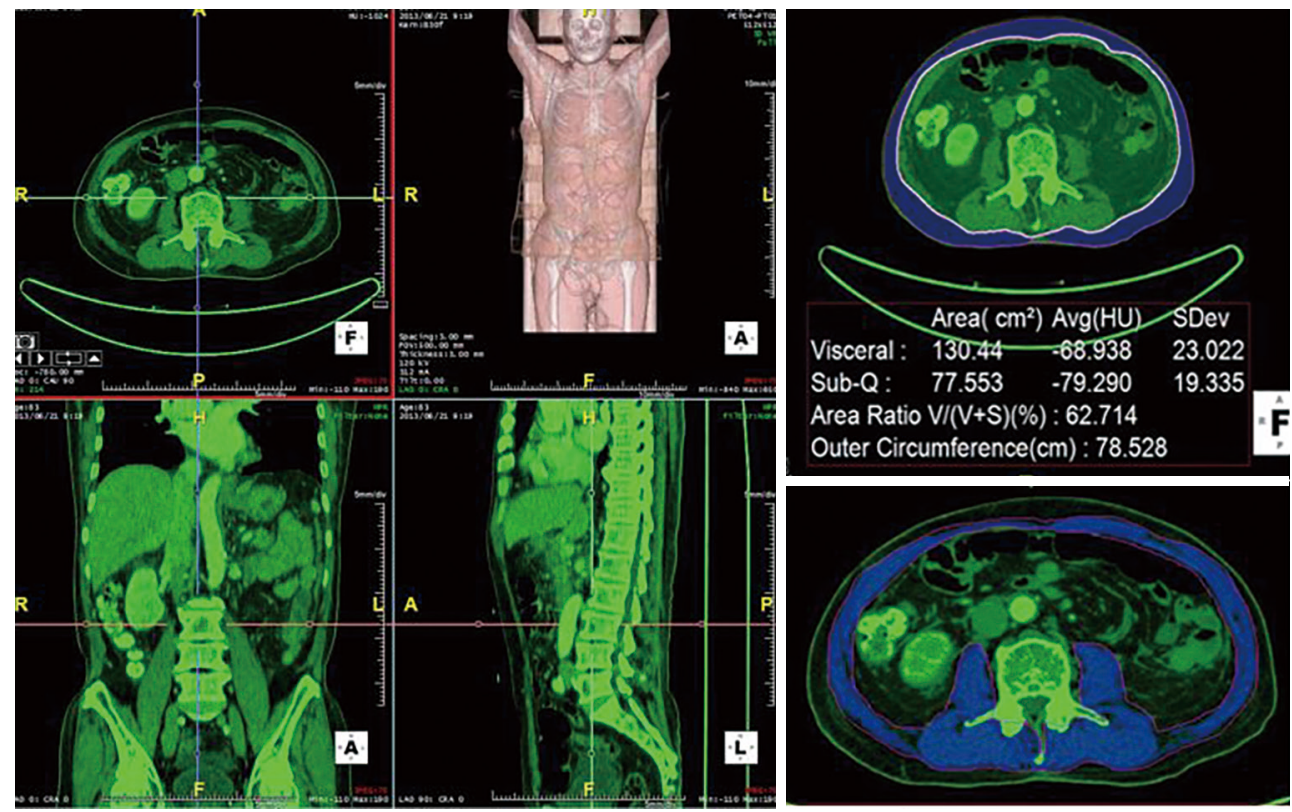

Figure 1 Muscle and fat mass measurement in PD patients. PD, pancreaticoduodenectomy.

and $\mathrm{C}$-reactive protein (CRP)-were measured at the preoperative period and on POD 7. All the indicators in the preoperative period were examined one day before surgery. NRI was calculated as follows; NRI $=(1.519 \times$ serum albumin, $\mathrm{g} / \mathrm{dL})+[41.7 \times$ present weight $(\mathrm{kg}) /$ ideal body weight $(\mathrm{kg})]$. Muscle density and muscle, visceral fat, and subcutaneous fat masses were determined using a CT scan (12) before surgery and POD 7 at the $3^{\text {rd }}$ lumbar spine (L3) level (Figure 1). The preoperative CT scan was taken at the out-patient clinic, and the average period from the day when the CT scan was taken to surgery was $16.25 \pm 5.78$ days. The CT scan was obtained using a 16- or 64-section scanner (Somatom Sensation 16 or 64; Siemens Medical Solutions, Forchheim, Germany) and a section thickness $3 \mathrm{~mm}$ (14).

To see whether they are affected by the inflammation, we analyzed the correlation between albumin and prealbumin, CRP and prealbumin. And the comparison of prealbumin to CRP ratio between preoperative period and POD 7 was conducted.

The difference of serum CRP level between the perioperative period and POD 7 was divided into three groups: (I) less than zero square of ten $\left(<10^{\circ}\right)$, (II) from greater than or equal to a square of ten up to less than two square of ten $\left(\leq 10^{1}-<10^{2}\right)$, (III) greater or equal to two square of ten $\left(\geq 10^{2}\right)$. For each group, we analyzed the correlation with prealbumin, muscle mass, muscle density, visceral and subcutaneous fat.
The study was conducted in accordance with the Declaration of Helsinki (as revised in 2013). The study was approved by institutional of CR321018 and informed consent was taken from all the patients

\section{Statistical analysis}

The statistical analysis was performed using SPSS version 23, and correlation analysis using the Pearson test. The paired $t$-test and ANOVA were used for group comparisons. Results are presented as means \pm SDs and statistical significance was accepted for $\mathrm{P}$ values $<0.05$.

\section{Results}

The study subjects were 18 males and 10 females of overall average age $63.5 \pm 9.7$ years, and BMI $22.89 \pm 3.11 \mathrm{~kg} / \mathrm{m}^{2}$. Of the 28 patients, common bile duct cancer was the most common diagnosis $(46.4 \%)$ following in decreasing order by ampulla of Vater cancer, pancreatic head cancer, chronic pancreatitis, and neuroendocrine tumor (Table 1).

NRI, protein, albumin, and prealbumin were significantly lower $(\mathrm{P}<0.001)$, but $C R P$ was significantly higher $(\mathrm{P}<0.001)$ at POD 7 than preoperative period (Table 2).

Prealbumin and albumin levels were positively correlated $(\mathrm{P}=0.006)$ (Figure 2). But on the contrary prealbumin and CRP levels were negatively correlated $(\mathrm{P}<0.001)$ (Figure 3$)$, 
and prealbumin to CRP ratio was significantly lower on POD $7(\mathrm{P}=0.003)$ (Figure 4). There was a significant difference in comparison the group divided by CRP change with prealbumin between the preoperative period and POD $7(\mathrm{P}<0.001)$ (Figure 5).

Muscle mass was higher $(\mathrm{P}=0.02)$ and visceral fat was lower $(\mathrm{P}<0.001)$ on POD 7 than preoperative period, but muscle density and amount of subcutaneous fat were similar in both periods (Figure 6). There was no significant difference in comparison between the groups divided by CRP change and muscle density, muscle mass, visceral and subcutaneous fat amount (Figure 7).

\section{Discussion}

Hepatobiliary and pancreatic malignancy is often associated with malnutrition, which is attributed to sustained pro-

Table 1 Patient characteristics

\begin{tabular}{lc}
\hline & Value \\
\hline Pancreaticoduodenectomy & 28 \\
Number & $18 / 10$ \\
Sex (male/female) & $63.54 \pm 9.72$ \\
Age & $22.89 \pm 3.11$ \\
BMI $\left(\mathrm{kg} / \mathrm{m}^{2}\right)$ & \\
Diagnosis & $13(46.4)$ \\
Common bile duct cancer & $9(32.1)$ \\
Ampulla of Vater cancer & $2(7.2)$ \\
Pancreas head cancer & $2(7.2)$ \\
Chronic pancreatitis & $2(7.2)$ \\
Neuroendocrine tumor &
\end{tabular}

inflammatory cytokine response, poor dietary intake, and the catabolic effects of sepsis $(15,16)$. As malnutrition has a negative effect on prognosis after radical surgery, adequate nutritional support is mandatory.

Various methods have been used to provide patients with sufficient nutrition during the postoperative period. The early recovery after surgery (ERAS) protocol reported that the initiation of early enteral feeding has shown to reduce complications and hospital stays, and promote earlier bowel function recovery and the resumption of normal activities $(17,18)$.

However, the anatomical change of the gastrointestinal tract after surgery causes an insufficient oral intake, an additional nutritional supply is required such as enteral tube feeding or TPN to provide enough calorie (6). The optimal means of parenteral feeding remains controversial and though numerous studies have compared the efficacy of enteral feeding and TPN, the results vary (19-23).

Various serologic parameters have been used to estimate the nutritional statuses of patients at the postoperative period. But, these parameters are easily influenced by environmental factors, inflammatory conditions, and drugs (10). As shown in Figures 3-5, our results verify this phenomenon. Therefore, it appears these parameters are not suitable to assess the nutritional status at immediate postoperative period because of inflammatory process in the body.

Sarcopenia is a syndrome characterized by progressive, generalized loss of skeletal muscle mass and strength, and can be diagnosed by low muscle mass and poor muscle function. Numerous studies have reported that sarcopenia is highly associated with poor outcomes, and mortality (24-30).

Recently, sarcopenia has been identified as a poor prognostic factor for patients with pancreatic cancer, colorectal with liver metastasis, liver cirrhosis and liver

Table 2 Postoperative nutritional marker changes

\begin{tabular}{lccc}
\hline & Preoperative & Postoperative day 7 & P \\
\hline BMI $\left(\mathrm{kg} / \mathrm{m}^{2}\right)$ & $22.98 \pm 3.03$ & $22.78 \pm 3.35$ & 0.282 \\
$\mathrm{NRI}$ & $50.27 \pm 7.02$ & $48.81 \pm 7.32$ & 0.001 \\
Protein $(\mathrm{g} / \mathrm{dL})$ & $6.04 \pm 0.62$ & $5.38 \pm 0.52$ & $<0.001$ \\
Albumin $(\mathrm{g} / \mathrm{dL})$ & $3.53 \pm 0.52$ & $2.84 \pm 0.31$ & $<0.001$ \\
Prealbumin (mg/L) & $166.87 \pm 68.54$ & $123.72 \pm 48.36$ & $<0.001$ \\
CRP $(\mathrm{mg} / \mathrm{dL})$ & $12.23 \pm 19.69$ & $74.33 \pm 48.85$ & $<0.001$ \\
\hline
\end{tabular}

$\mathrm{BMI}$, body mass index; NRI, nutritional risk index; CRP, C-reactive protein. 

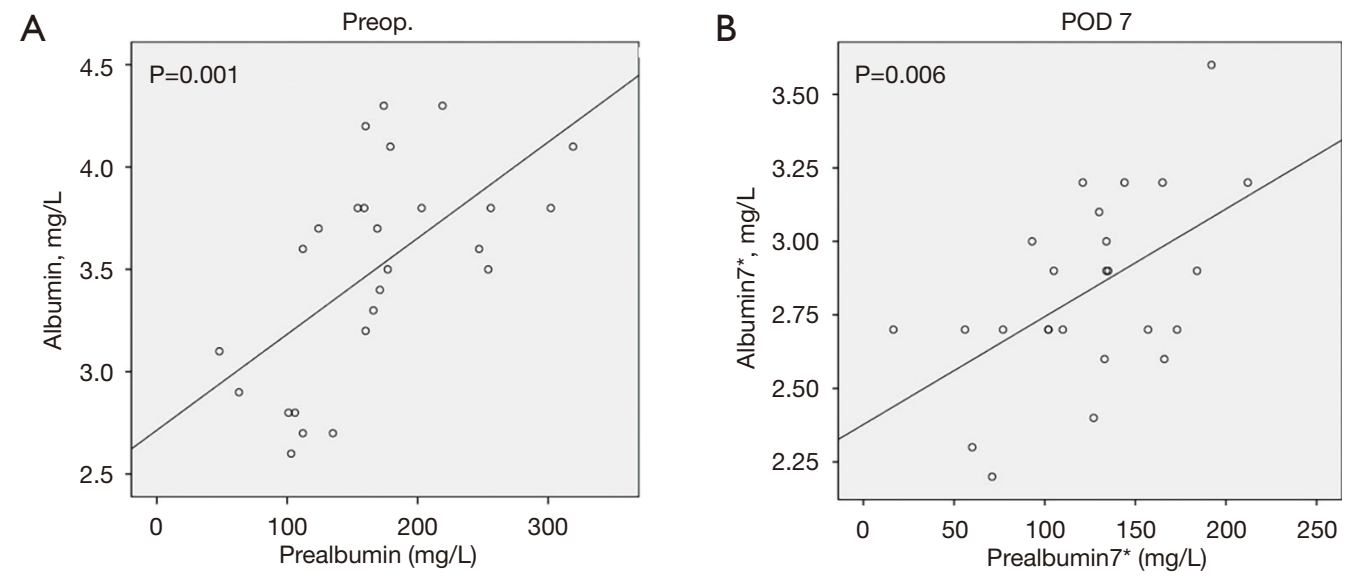

Figure 2 Correlations between prealbumin and albumin (A), and prealbumin and CRP (B) levels. *, Albumin 7 \& Prealbumin 7 , serum albumin and prealbumin level on POD 7; Preop, preoperative period; POD 7, postoperative day 7; CRP, C-reactive protein.
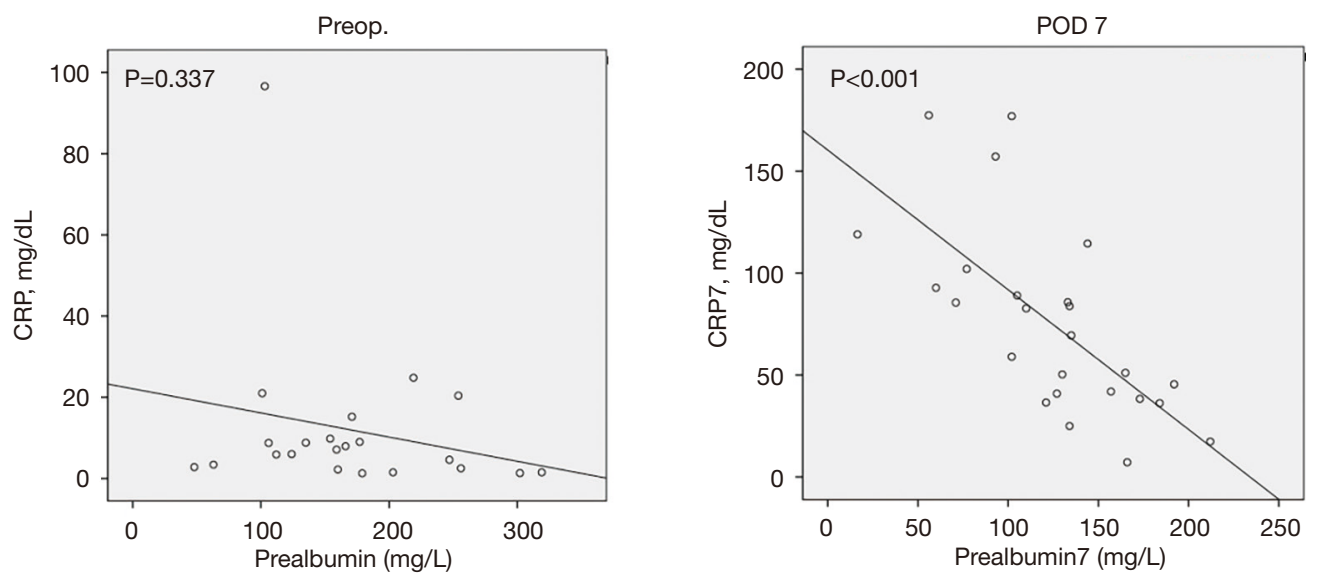

Figure 3 Postoperative changes in prealbumin-CRP ratios. CRP, C-reactive protein.
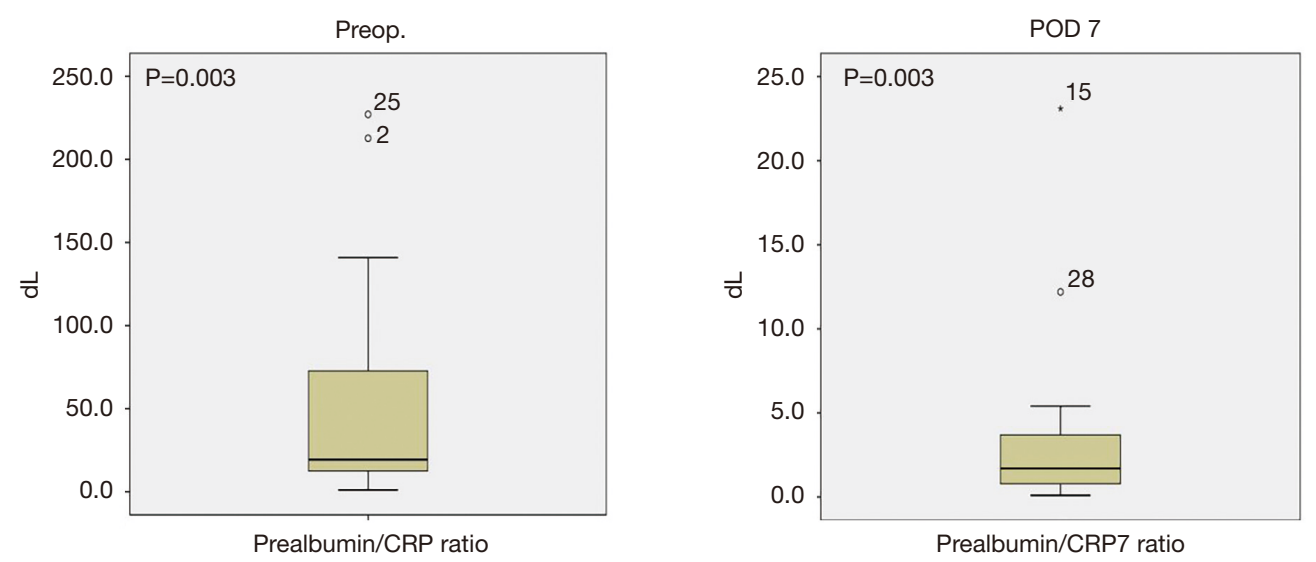

Figure 4 Comparison of prealbumin/CRP ratio. CRP, C-reactive protein. 

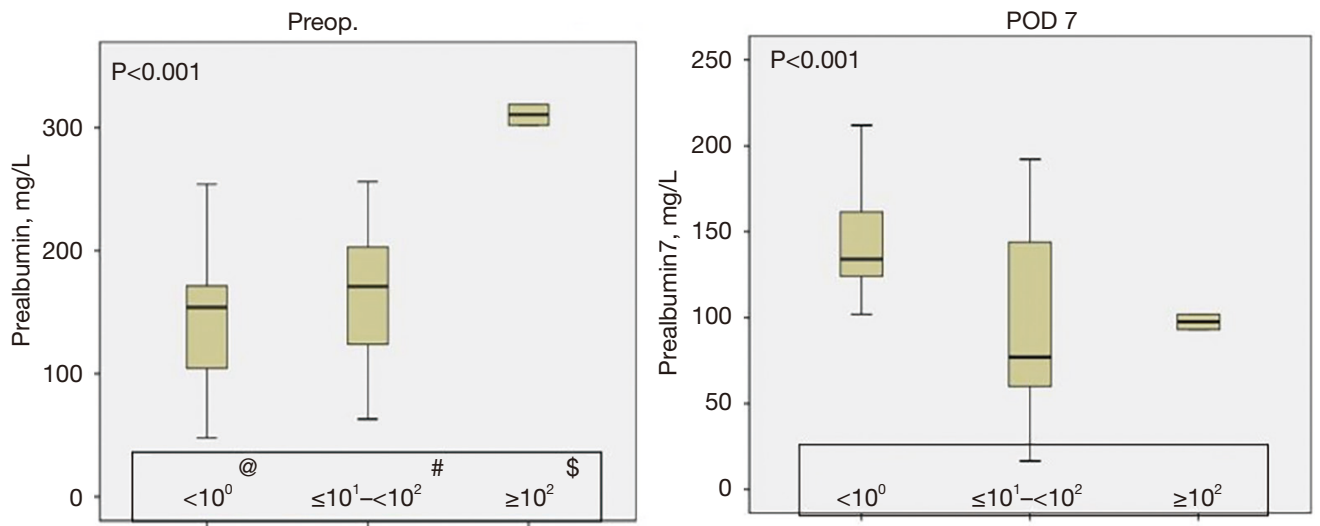

Figure 5 Variation of Prealbumin level according to the changes of CRP. @, less than zero square of ten; \#, from greater than or equal to a square of ten up to less than two square of ten; \$, greater or equal to two square of ten. CRP, C-reactive protein.

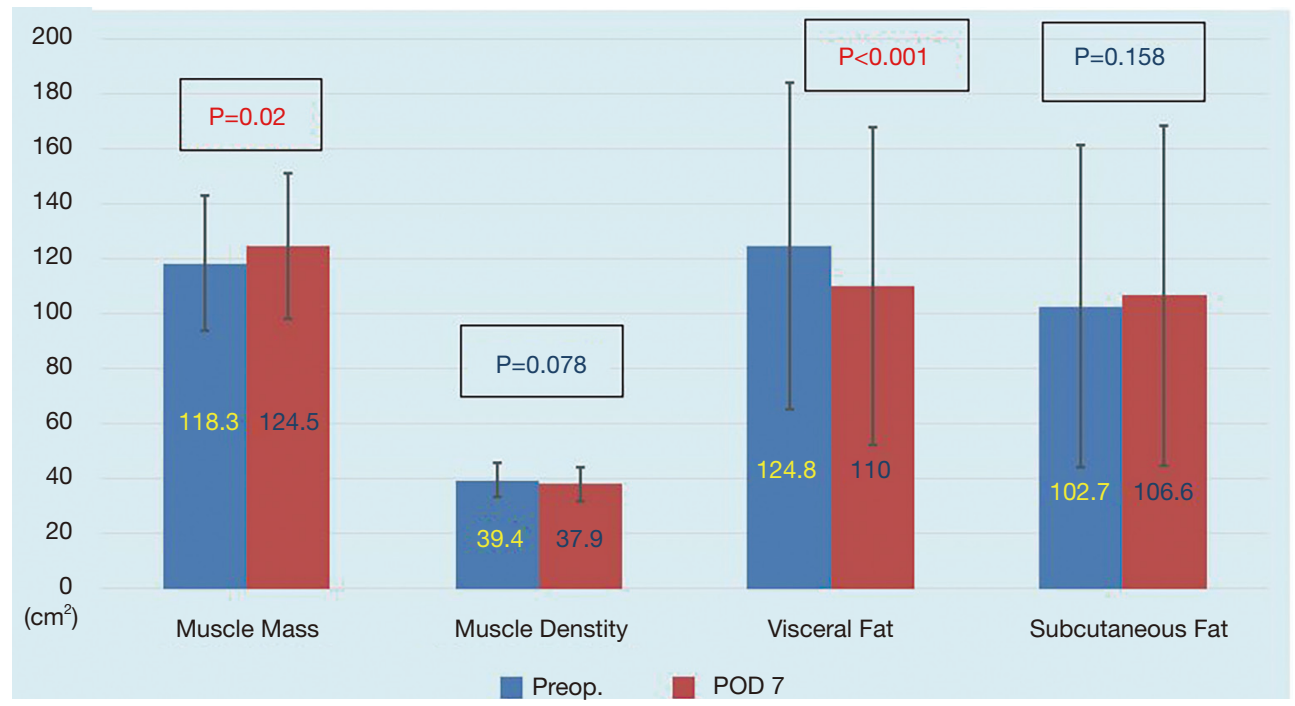

Figure 6 Comparison of anthropometric parameters. Muscle mass and density, visceral and subcutaneous fat amounts were evaluated by CT scan at L3 level, and compared between preoperative period and POD 7. POD7, postoperative day 7.

transplantation (31-34). Besides, sarcopenia is known to have negative impacts on postoperative morbidity and prognosis undergoing $\mathrm{PD}$, and also it is the risk factor for postoperative pancreatic fistula which is the most fatal complication after PD (34-36). Therefore, prevention of sarcopenia after surgery is important.

Several methods can be used to assess muscle mass, but all have advantages and disadvantages. Although CT scan measures of muscle mass provide the most reliable and accurate information, the technique is hospitalbased, expensive, and time-consuming as compared with other modalities. But, as all the patients who underwent
PD routinely take CT scan postoperatively to check complications, the anthropometric data can be collected without an additional charge.

To determine the effectiveness of TPN, we compared muscle mass, muscle density, and visceral and subcutaneous fat amounts measured preoperatively and on POD 7. Our important finding was that although visceral fat amounts were reduced on POD 7, muscle mass was unaffected or even tended to increase, which can be explained that the protein sparing effect of TPN administration prevented muscle degradation. Furthermore, the maintenance or increase of muscle mass can prevent sarcopenia by 

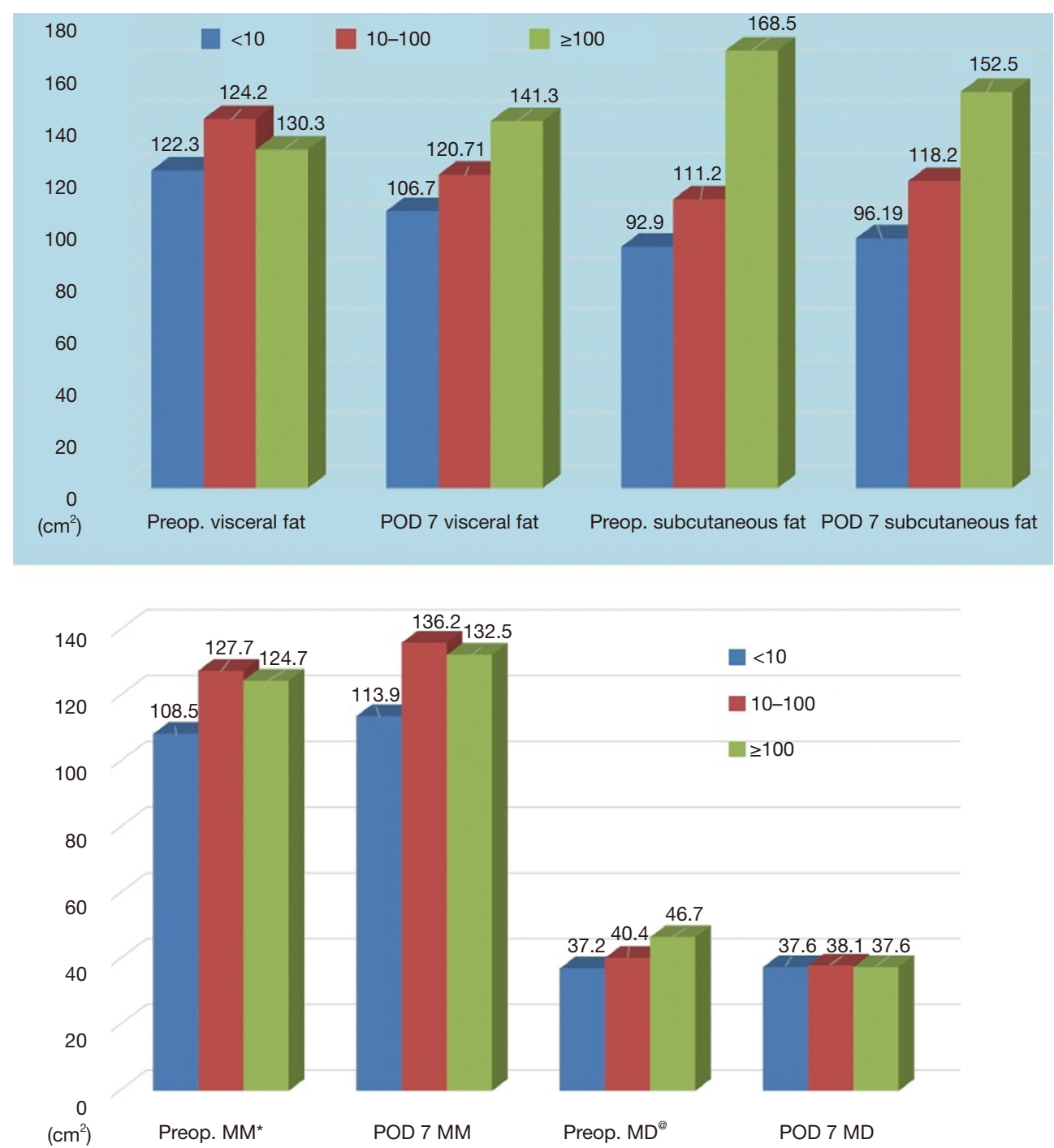

Figure 7 Changes in muscle mass, muscle density, visceral and subcutaneous fat amounts with respect to CRP. *, muscle mass; @, muscle density; CRP, C-reactive protein.

appropriate TPN administration.

In order to evaluate the nutrition status accurately after $\mathrm{PD}$, we suggest that CT scan-based measurement of muscle and fat mass measurements at the postoperative period. Also we suggest a prospective study to compare nutritional statuses in enteral feeding and TPN groups after PD and to investigate the effects of oral intake amounts and the timing of supplementation.

The limitations of this study include a small number of patient populations and the lack of similar research related to the effectiveness of TPN at early postoperative period after major surgery.

\section{Acknowledgments}

Funding: None.

\section{Footnote}

Reporting Checklist: The authors have completed the STROBE reporting checklist. Available at https://dx.doi. org/10.21037/gs-20-390

Data Sharing Statement: Available at https://dx.doi. org/10.21037/gs-20-390 
Conflicts of Interest: All authors have completed the ICMJE uniform disclosure form (available at https://dx.doi. org/10.21037/gs-20-390). The authors have no conflicts of interest to declare.

Ethical Statement: The authors are accountable for all aspects of the work in ensuring that questions related to the accuracy or integrity of any part of the work are appropriately investigated and resolved. The study was conducted in accordance with the Declaration of Helsinki (as revised in 2013). The study was approved by institutional review board of CR321018 and informed consent was taken from all the patients.

Open Access Statement: This is an Open Access article distributed in accordance with the Creative Commons Attribution-NonCommercial-NoDerivs 4.0 International License (CC BY-NC-ND 4.0), which permits the noncommercial replication and distribution of the article with the strict proviso that no changes or edits are made and the original work is properly cited (including links to both the formal publication through the relevant DOI and the license). See: https://creativecommons.org/licenses/by-nc-nd/4.0/.

\section{References}

1. Whipple AO, Parsons WB, Mullins CR. Treatment of carcinoma of the ampulla of Vater. Ann Surg 1935;102:763-79.

2. Kang CM, Lee JH. Pathophysiology after pancreaticoduodenectomy. World J Gastroenterol 2015;21:5794-804.

3. Wastell C. Malabsorptive states after gastrointestinal surgery. Br Med J 1968;3:661-4.

4. Alberino F, Gatta A, Amodio P, et al. Nutrition and survival in patients with liver cirrhosis. Nutrition 2001;17:445-50.

5. Worsh CE, Tatarian T, Singh A, et al. Total parenteral nutrition in patients following pancreaticoduodenectomy: lessons from 1184 patients. J Surg Res 2017;218:156-61.

6. Braga M, Gianotti L, Vignali A, et al. Artificial nutrition after major abdominal surgery: impact of route of administration and composition of the diet. Crit Care Med 1998;26:24-30.

7. Dudrick SJ, Wilmore DW, Vars HM, et al. Long-term total parenteral nutrition with growth, development, and positive nitrogen balance. Surgery 1968;64:134-42.

8. Everitt NJ. Enteral nutrition is superior to parenteral nutrition in severe acute pancreatitis: results of a randomized prospective trial. Br J Surg 1998;85:716.

9. Heyland DK, MacDonald S, Keefe L, et al. Total parenteral nutrition in the critically ill patient: a metaanalysis. JAMA 1998;280:2013-9.

10. Bharadwaj S, Ginoya S, Tandon P, et al. Malnutrition: laboratory markers vs nutritional assessment. Gastroenterol Rep (Oxf) 2016;4:272-80.

11. Beck FK, Rosenthal TC. Prealbumin: a marker for nutritional evaluation. Am Fam Physician 2002;65:1575-8.

12. Rubbieri G, Mossello E, Di Bari M. Techniques for the diagnosis of sarcopenia. Clin Cases Miner Bone Metab 2014;11:181-4.

13. Braga $M$, Ljungqvist $O$, Soeters $P$, et al. ESPEN Guidelines on Parenteral Nutrition: surgery. Clin Nutr 2009;28:378-86.

14. Kim KE, Park MS, Bentley-Hibbert S, et al. Hepatocellular carcinoma: clinical and radiological findings in patients with chronic $\mathrm{B}$ viral hepatitis and chronic C viral hepatitis. Abdom Imaging 2012;37:591-4.

15. Fearon KC, Barber MD, Falconer JS, et al. Pancreatic cancer as a model: inflammatory mediators, acutephase response, and cancer cachexia. World J Surg 1999;23:584-8.

16. Braga M, Gianotti L, Nespoli L, et al. Nutritional approach in malnourished surgical patients: a prospective randomized study. Arch Surg 2002;137:174-80.

17. Pruthi RS, Nielsen $M$, Smith A, et al. Fast track program in patients undergoing radical cystectomy: results in 362 consecutive patients. J Am Coll Surg 2010;210:93-9.

18. Durkin MT, Mercer KG, McNulty MF, et al. Vascular surgical society of great britain and ireland: contribution of malnutrition to postoperative morbidity in vascular surgical patients Br J Surg 1999;86:702.

19. Guilbaud T, Birnbaum DJ, Loubière $S$, et al. Comparison of different feeding regimes after pancreatoduodenectomy - a retrospective cohort analysis. Nutr J 2017;16:42.

20. Probst P, Keller D, Steimer J, et al. Early combined parenteral and enteral nutrition for pancreaticoduodenectomy - Retrospective cohort analysis. Ann Med Surg (Lond) 2016;6:68-73.

21. Perinel J, Mariette C, Dousset B, et al. Early Enteral Versus Total Parenteral Nutrition in Patients Undergoing Pancreaticoduodenectomy: A Randomized Multicenter Controlled Trial (Nutri-DPC). Ann Surg 2016;264:731-7.

22. Mueller MH, Vandenbussche K, Pelliccia M, et al. Enteral Nutrition Support Reduces the Necessity of Total Parenteral Nutrition to Reach Patient-Specific Caloric Goals Postpancreaticoduodenectomy. South Med J 
2015;108:748-53.

23. Cereda E, Caccialanza R, Pedrolli C. Feeding after pancreaticoduodenectomy: enteral, or parenteral, that is the question. J Thorac Dis 2016;8:E1478-80.

24. Friedman J, Lussiez A, Sullivan J, et al. Implications of sarcopenia in major surgery. Nutr Clin Pract 2015;30:175-9.

25. Kudou K, Saeki H, Nakashima Y, et al. Prognostic Significance of Sarcopenia in Patients with Esophagogastric Junction Cancer or Upper Gastric Cancer. Ann Surg Oncol 2017;24:1804-10.

26. Miller JA, Harris K, Roche C, et al. Sarcopenia is a predictor of outcomes after lobectomy. J Thorac Dis 2018;10:432-40.

27. Du Y, Karvellas CJ, Baracos V, et al. Sarcopenia is a predictor of outcomes in very elderly patients undergoing emergency surgery. Surgery 2014;156:521-7.

28. Mayr R, Fritsche HM, Zeman F, et al. Sarcopenia predicts 90-day mortality and postoperative complications after radical cystectomy for bladder cancer. World J Urol 2018;36:1201-7.

29. Smith A. Sarcopenia, malnutrition and nutrient density in older people. Post Reprod Health 2014;20:19-21.

30. Zhou CJ, Zhang FM, Zhang FY, et al. Sarcopenia: a new

Cite this article as: Cha SW, Kim SH, Baek SE, Kim KS. Development of a nutritional index to evaluate the effectiveness of total parenteral nutrition during the early postoperative period after pancreaticoduodenectomy. Gland Surg 2021;10(9):2622-2630. doi: 10.21037/gs-20-390 predictor of postoperative complications for elderly gastric cancer patients who underwent radical gastrectomy. J Surg Res 2017;211:137-46.

31. Montano-Loza AJ, Meza-Junco J, Prado CM, et al. Muscle wasting is associated with mortality in patients with cirrhosis. Clin Gastroenterol Hepatol 2012;10:166-173.e1.

32. Prado CM, Lieffers JR, McCargar LJ, et al. Prevalence and clinical implications of sarcopenic obesity in patients with solid tumours of the respiratory and gastrointestinal tracts: a population-based study. Lancet Oncol 2008;9:629-35.

33. Englesbe MJ, Patel SP, He K, et al. Sarcopenia and mortality after liver transplantation. J Am Coll Surg 2010;211:271-8.

34. Tan BH, Birdsell LA, Martin L, et al. Sarcopenia in an overweight or obese patient is an adverse prognostic factor in pancreatic cancer. Clin Cancer Res 2009;15:6973-9.

35. Peng P, Hyder O, Firoozmand A, et al. Impact of sarcopenia on outcomes following resection of pancreatic adenocarcinoma. J Gastrointest Surg 2012;16:1478-86.

36. Joglekar S, Asghar A, Mott SL, et al. Sarcopenia is an independent predictor of complications following pancreatectomy for adenocarcinoma. J Surg Oncol 2015;111:771-5. 